\title{
Photo-Curing Schemes to Cure the Epoxy Resins and their Impacts on Curing Process
}

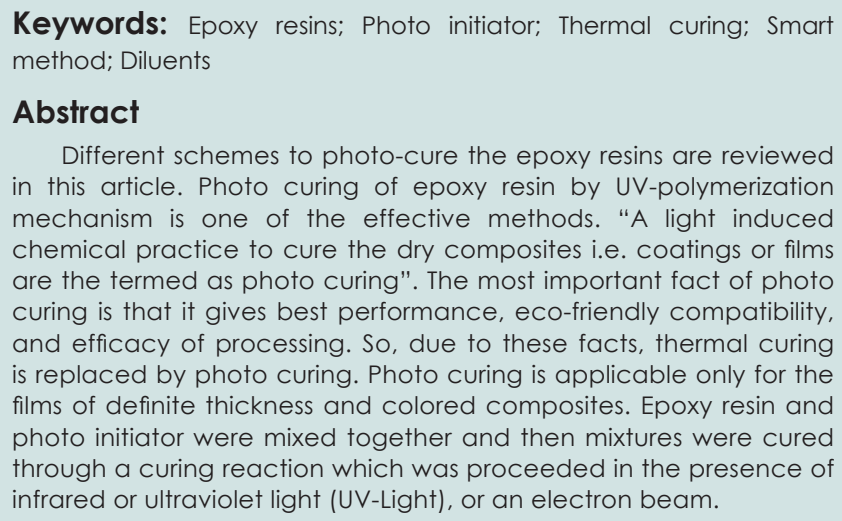

Keywords: Epoxy resins; Photo initiator; Thermal curing; Smart method; Diluents

\begin{abstract}
Different schemes to photo-cure the epoxy resins are reviewed in this article. Photo curing of epoxy resin by UV-polymerization mechanism is one of the effective methods. "A light induced chemical practice to cure the dry composites i.e. coatings or films are the termed as photo curing". The most important fact of photo curing is that it gives best performance, eco-friendly compatibility, and efficacy of processing. So, due to these facts, thermal curing is replaced by photo curing. Photo curing is applicable only for the films of definite thickness and colored composites. Epoxy resin and photo initiator were mixed together and then mixtures were cured through a curing reaction which was proceeded in the presence of infrared or ultraviolet light (UV-Light), or an electron beam.
\end{abstract}

\section{Introduction}

Photo curing of ER by UV-polymerization mechanism is one of the effective methods. "A light induced chemical practice to cure the dry composites i.e. Coatings or films are the termed as photo curing". The most important fact of photo curing is that it gives best performance, eco-friendly compatibility, and efficacy of processing. So, due to these facts, thermal curing is replaced by photo curing. Photo curing is applicable only for the films of definite thickness and colored composites. It efficiently works on the thin films of composites. Photo curing also reduces some basic problems occurring in the different methods of curing like curing on one line of sight, absorption of light in thick film and coloring agents. Thermal curing demands the corresponding facility of air-drying, and also pollutes the environment. But photo curing is efficient method because it has less these problems rather it solves them. It has none of solvent for evaporation. It does not pollute the environment and loses the coatings in very limited amount. Its results appear in very short time period and it causes very less pollution in environment. Also the photo curing brings several outstanding manual characteristics such as marvelous scratch, resistance against corrosion, and excellent bonding properties [1].

An UV curing is a surface treatment which either is cured by ultraviolet radiation, or which protects the underlying material from such radiation's harmful effects. UV-curing technology has been popular for decades due to its significant advantages including lower energy consumption, less environmental pollution lower process costs, excellent film quality, high efficiency in production, fastness of process and no oxygen inhibition [2]. It also has a wide range of applications such as coatings, inks, adhesives, and composite materials [3].

Photo cured ER have great attention due to the excellent properties and outstanding working performance. ER also has 3-dimensional stabilities, adhesion properties, and efficient resistance against corrosion [4]. They are applicable in several industries including the floor making, laminate production, aerospace, and aircraft [5].
Journal of

Chemistry \& Applications

\section{Fazal-ur-Rehman}

Department of Chemistry, University of Education, Lahore-Vehari Campus, Pakistan

\section{Address for Correspondence}

M. Fazal-ur-Rehman, Department of Chemistry, University of Education, Lahore-Vehari Campus, Punjab, Pakistan, Tel: +923467088517; E-mail: fazalurrehman517@gmail.com

Submission: 04 June, 2018

Accepted: 23 July, 2018

Published: 27 July, 2018

Copyright: (๑ 2018 Fazal-ur-Rehman M. This is an open access article distributed under the Creative Commons Attribution License, which permits unrestricted use, distribution, and reproduction in any medium, provided the original work is properly cited.

To enhance their properties, some nanofillers e.g. Clay alumina, titania, colloidal silica, and carbon nano tubes, were added. To obtain most beneficial characteristics, EC are cured by heat or radiations. This is an excellent method to cure EC but have a problem that radiation hindered due to color additives [6].

Different schemes to photo-cure the ER are detailed here.

\section{Scheme 1}

Carbon black (CB), Azo-Yellow and Phthalocyanine green have been utilized as commercial fillers. Monomer of 1,4-butanediol diglycidyl ether was used. PI of Triarylsulfonium hexafluoroantimonate salt in 50\% wt within propylene carbonate was taken. The samples were prepared by two methods; i. Conventional method. ii. Smart method.

Conventional method: Filler mixture, PI and Monomer was passed through process of preparation, irradiation then poured into molds. The prepared samples were stored at $296 \mathrm{~K}$ for period of 12 hours. The Standard sample was also prepared by adopting the same method.

Smart method: PI solution was passed through irradiation and then mixing with filler and epoxy in relative concentration. Then mixture was poured into mold and kept at $296 \mathrm{~K}$ for period of 12 hours. The Standard sample was also prepared by adopting the same method [7].

\section{Scheme 2}

Tertiary tetramine which is being functionalized with six allyl groups is used as curing agent of epoxy composites. It is anionically polymerized for the production of polyether. Curing agents also have double bonds which react with the thiol and form polythioethers. The unreacted composites can be completely polymerized by polyethers. Modulus of 25000-29000 mpa is used to cure the specimens completely [8].

\section{Scheme 3}

If graphene is being dispersed in any polymeric resin it will ultimately enhances is mechanical properties. For verification, antiscratch test is used. For curing of ER, reduced graphene is investigated 
which is being enhanced by amino functionalized organo-silanes. The results revealed that by adding fingorin ER, their mechanical properties becomes high and their ability to form strong covalent bond increases a lot. Materials are taken and fillers are prepared. Coating is also prepared and tests are done to investigate the effectiveness of graphene against ER. Fngoris tested with GO, GNP and amino fnsiO $\mathrm{O}_{2}$ reinforced cycloaliphatic ER. Conclusion has shown the effectiveness of fngor [9].

\section{Scheme 4}

Jin et al. reviewed the synthesis and curing process of ER. Also they discussed the method of preparation and applications of ER.

Curing agents/processes: Different curing processes are used to cure ER. One of the main agents is epoxy curing agents. By the addition of these agents the ER can be cured. Other processes include amine type curing agents, alkali curing agents, anhydride curing agents and catalytic curing agents.

Curing systems: Different curing systems are discussed which can cure ER by the reacting epoxide groups in ER. And it forms a complex cross-linked, 3-D network. There are different curing systems which includes room-temperature curing, heat curing and photo-curing. Photo-curing is the best technique among all of them. It involves infrared, UV and electron beam irradiation. And it reduces the curing time from hours to minutes.

Epoxy based composites: Epoxy based composites are rigid and brittle and have lower resistance towards growth and initiation. It ultimately limits the use of ER in many applications. And to overcome this issue different ER are prepared which includes thermoplastic modified epoxy system, epoxy/inorganic composites, epoxy/carbon fiber composites, epoxy/clay nano composites and epoxy/carbon nano tube composites [3].

\section{Scheme 5}

Shen et al. used variable monomers and oligomers and coated them with temporary and permanent coating. Also a series of UV curable hydrophilic resins were used results have shown that the cured coatings have alkaline ability in them. Samples are taken, synthesized and cured. In curing of resins, the coated formulations were cured at room temperature by equipment named Lab Cure UV tunnel. After this process the films and other specimens are kept at ambient overnight for characterization. Then samples are characterized by FTIR spectra, NMR spectra, GPC measurements, chemical and water resistance, contact angle measurements, SEM, DSC measurements, TGA analysis and photo polymerization mechanisms [2].

\section{Scheme 6}

M Atif et al. used thick or thin films as transparent or colored composites for UV-curing. Thin films are preferred as their mechanical performance is excellent, thermally stable compounds and have unique electrical or optical properties. Thick films are also used to be cured because of its property to lessen the fabrication expenses of complex assemblies. This technique results in the formation of liquid monomer (solvent free) into complex inter-linked polymer (at room temperature). Thick samples cannot be cured properly as light cannot penetrate into them. Also, little curing takes place when colored additives or filers are present. With the new inventions these issues can be resolved. Cationic polymerization mechanism is used which is very complicated and have many steps.
Initiation involves the stimulation of onium salts. The salts then undergo cleavage and cations and aryl cations are produced. In this way, it produces strong Bronsted acids which are used as starting material of polymerization. After this steps, reaction again takes place and from secondary onium ion.

Propagation involves two mechanisms: 1 . Activated chain end (ACE) mechanism, 2. Activated monomer (AM) mechanism. In ACE mechanism, when the onium ton is being produced, it propagates to grow the chain. In AM mechanism, during polymerization, the ionic end of the growing chain is encountered by the alcohol molecule by which the protonated ether is being furnished. So, by these two mechanisms the chain is propagated.

Termination includes different processes. In the propagation chain the nucleophile impurities e.g. water get interacted with it and release inactive species due to which the chain terminates. The chain is also terminated by chain transfer reactions [10].

\section{Scheme 7}

$10 \mathrm{mg}$ of Graphene sheets (GNP) is reacted to $10 \mathrm{ml}$ of $\mathrm{H}_{2} \mathrm{O} /$ etoh $50 \%$. This is done in ultra-sonication bath. After that $\mathrm{KaUCl}_{4}$ is added to concentrate the dispersion of about $5 \mathrm{~mm}$. When the deposition comes to an end, particles are being washed and dried up for a night at $70{ }^{\circ} \mathrm{C}$ to obtain neutral PH. Then Au-GNP is dispersed in the epoxy resin, ranging $0.5-2.0 \mathrm{wt} \%$. This is done with the help of ultraturrax $0.2 \mathrm{wt} \%$ of PI was added after mixing. Different methods are used to determine the extent of photopolymerization. It includes Real-TimeFTIR spectroscopy, ASTM D2765-84 and DMTA [11].

\section{Scheme 8}

Different types of phosphate esters were prepared. This may include butyl phosphate ester (BPE), ethyl phosphate ester (EPE) etc. These were prepared by the ratio 2:1. ER and each of the phosphate ester is mixed together to form uniform mixture. Then by NMR, CCT, and TGA tests the specimens were examined and phosphate esters were formed [12].

\section{Scheme 9}

Two samples of glass fiber reinforced epoxy (GRE) composites were prepared and polymerized by VPA monomers. Their flammability was tested by cone calorimetric test (generally called as fire test). Results showed that samples with thicker coatings did not catch fire. Also thicker coated samples were resistant to peel off while come in contact with tap pull test. But they lost its coating in water soaked test showing its hydrophobicity [13].

\section{Scheme 10}

The following materials were used to photo cure the epoxy composites; Cycloaliphatic ER, 3,4-epoxycyclohexaylmethyl 3,4-epoxy cyclohexane carboxylate (CE) and triphenyl sulphonium hexaflouroantimonate as cationic PI, nanoparticles of $\alpha-\mathrm{Al}_{2} \mathrm{O}_{3}$ with $150 \mathrm{~nm}$ diameter and specific surface area of $14.5 \mathrm{~m}^{2} / \mathrm{g}$. Several amounts of Alumina particles with volume range of $0.5 \%$ and $0.4 \%$ were allowed to dispersed in epoxy composite via an ultrasonic water bath for period of 120 minutes and then passed through ultra turrax for period of 5 minutes at $25000 \mathrm{rpm}$. Then all mixtures with cationic PI ( $2 \mathrm{wt} \%$ ) were added and cured under static UV-lamp whose having intensity of $55 \mathrm{mw} / \mathrm{cm}^{2}$ at surface of sample [14]. 


\section{Scheme 11}

An ER named as 1,6-hexanediol diglycidyl ether (HDGE) in weightage of 50, 60, 70 \& $75 \mathrm{wt} \%$ was mixed with Zinc epoxy to prepare the homogeneous mixture. An ultra turrax was used for period of 10 minutes at the speed of $30000 \mathrm{rpm}$ to achieve the chemically homogenous composition and dispersion of zinc oxide powder. After that, PI in $4 \mathrm{wt} \%$ was dropped in mixture and then mixture was placed at ultrasonic bath for the period of 20 minutes. Coating of PI was appeared at mixture formulations between substrates of $2 \mathrm{PP}$. Then it was passed through curing via UV-rays of intensity $40 \mathrm{mw} . \mathrm{cm}^{2}$ for 5 minutes [15].

\section{Scheme 12}

The mixture of epoxy resins with $\mathrm{Gd}_{2} \mathrm{O}_{3}: \mathrm{Eu}^{3+}$ nanorods were prepared by dispersing the nanorods into UV-curable resins. After that the sonication of mixture was done for about 60 minutes and then addition of cationic PI $(2 \mathrm{wt} \%)$ was proceeded. Then these formulations were passed through spinning at three different stages of $500 \mathrm{rpm}, 1000 \mathrm{rpm} \& 2000 \mathrm{rpm}$. Spinning produced coatings on glass slides of mixture. Then these were cured by UV-light (intensity of $50 \mathrm{mw} . \mathrm{cm}^{2}$ in air about 3 minutes [16].

\section{Scheme 13}

Clean samples of ER and nano composites with 0.25-0.75 wt\% were prepared by using epoxy photo-curable resin SOMOS 10220 (Multiacrylate monomers $40-50 \%, 15-25 \%$ epoxies, $20-35 \%$ polyols and 0.2-5.0 wt\% of PI). Then ER became a mixture of acrylate monomer and epoxy oligomer. Multiwalled carbon nano tubes (MWCNT) were dispersed by combined sonication-mechanical \& magnetic stirring. The sonication was proceeded about 24 minutes. This sonication period was divided into 16-cycles; each cycle was consisted of 90 seconds with the rest of 15 seconds to save the sample of $40 \mathrm{~g}$ epoxy from overheating. At that time, sample was stirred mechanically and magnetically also. Samples of width $0.5 \pm 1.0$ were photo-cured for period of 12 and 24 hours by UV-A lamps of 355 $\mathrm{nm}$ and have $0.08 \mathrm{~W}-\mathrm{cm}^{-2}$ intensity at $313 \mathrm{k}$. After that samples were dispersed and analyzed via transmission electronic microscopy (TEM) in JOEL-JEM exii of $120 \mathrm{kv}$ instruments. Then samples were thermo-magnetically up to $873 \mathrm{k}$ at $283 \mathrm{k} / \mathrm{min}$ heating rate in a NETZSCH STA 449C instrument [17].

\section{Scheme 14}

The dispersion of carbon based nano fillers into ER was proceeded directly by a IKA Ultra turrax at $30000 \mathrm{rpm}$ for period of 10 minutes. Then samples were kept within ultrasonic bath for period of 10 minutes. Then $2 \%$ PI with respect to resin was added in carbon nano fillers. The coatings were disappeared on glass and samples were passed through photo-curing. The UV-irradiation was performed to develop the UVpolymerization, with $55 \mathrm{mw} / \mathrm{cm}^{2}$ light intensity on sample surface for duration of 15 minutes and then 3 minutes for transparency of resin. The coatings of homogenous disc shaped nano-composite (thickness of $150 \mu \mathrm{m}$ and diameter of $2 \mathrm{~cm}$ ) were appeared [18].

\section{Scheme 15}

An IKA Ultra turrax (30000 rpm) was used for period of 10 minutes to disperse the carbon base nano fillers in ER. After dispersion, all mixtures were kept in ultra-sonic bath. $2 \mathrm{wt} \%$ PI was mixed within the ER. Then glass substrates were covered by coatings of these mixtures and then cured by UV-Light. UV-irradiation with intensity of $55 \mathrm{mw} / \mathrm{cm}^{2}$ on sample surface, was done to promote the photo polymerization for duration of 15 minutes. After that, disc shaped homogenous coatings having $150 \mathrm{~mm}$ thickness and diameter of $2 \mathrm{~cm}$ were prepared and cut into small samples for DMTA and surface hardness analysis [19].

\section{Scheme 16}

Diglycidyl ether of bias (DGEBA) and ER equaling in amount was photo cured by a BOLTORN ${ }^{\circ}$ H40, hydroxyl terminated hyperbranched polyester (Mw $5100 \mathrm{~g} / \mathrm{mol}$ ) and 64 terminal groups. triarylsulfonium hexafluoroantimonate (TAS-Sb) was utilized as cationic PI. Different amounts of these materials were mixed and heated with hot air blower and stirred to prepare liquid mixtures of DGEBA and solid $\mathrm{H} 40.2 \%$ of PI was mixed within in samples to photo-cure. Then samples were passed through stirring and placing it at $255 \mathrm{k}$ to avoid the photo activation and photo polymerization. Photo curing of different samples were proceeded at several values of temperature. Samples were cured with mettler DSC-821e calorimeter which was modified by a Hamamatsu lightning cure LC5 (Hg-Xe Lamps) with two beams. From these, first beam was used for sample side while other was consumed for standard side. The curing of samples was proceeded in an open aluminum pans within nitrogenous atmosphere. Every sample was passed through scanning two times to disappear the UV-irradiation thermal effect. First time scan was performed about 4 minutes while irradiation was done about 30 minutes and then for 4 minutes in absence of UV-light. The light of $21 \mathrm{mw} / \mathrm{cm}^{2}$ intensity was used. Other dynamic post curing was performed in that DSC in absence of UV-Light for period of 10 minutes at 303-523k to find out the heat residual [20].

\section{Scheme 17}

An Ultra turrax was used at $30000 \mathrm{rpm}$ for duration of 10 minutes to disperse the $0.5-1.5 \mathrm{wt} \%$ graphene into ER. Then ultrasonic bath was also used for fine dispersion of fillers within ER. After that, triphenylsulfonium hexaflouroantimonate (2 wt\%) as PI was mixed in every sample. To perform DMTA and Surface hardness test, glass substrates was covered with coatings of these samples to cure through UV-light. UV-irradiation with intensity of $55 \mathrm{mw} / \mathrm{cm}^{2}$ on sample surface, was done to promote the photo polymerization for duration of 15 minutes. After that, disc shaped homogenous coatings having $25 \mathrm{~mm}$ thickness and diameter of $3 \mathrm{~cm}$ were prepared and cut into small samples [21].

\section{Scheme 18}

Monitoring of monomeric photo-polymerization was done by use of infrared spectroscopy [22]. The apparatus was adjusted with UVEXS Model SCU-110 mercury arc lamp (Sunnyvale, CA) which was fitted with flexible liquid optic wand. This wand was placed at angle of 450 at sample. While the distance between this apparatus and sample was altered to monitor the intensity of light. Intensities of UV-light were determined by UV-Supply procedure [23].

\section{Scheme 19}

The dispersion of CNTs (0.1 wt \%) within ER was proceeded. Then these were mixed by mechanical means of an ultra-turrax for 60 seconds. After mechanical mixing, thick layers $(200 \mathrm{~nm})$ were 
produced. These layers were cut by using a Reichert Ultracut S Ultrasmicrotome at 298k. Cutting device used to cut these layers was a diamond knife having cutting angle of 358 and dub [24].

\section{Scheme 20}

Three types of patterns are used. Pattern I: In this scheme, direct photo induced polymerization reactions concern the creation of a polymer through a chain reaction initiated by light. Pattern II: Since direct formation of reactive species on the monomer by light absorption is not an efficient route, the initiation step of the polymerization reaction requires the presence of a PI which, under light excitation, is capable of generating these reactive species. Pattern III: Extension of the spectral sensitivity (that corresponds to the best matching between the emission spectrum of the light source and the absorption spectrum of the formulation) can be achieved by using (pss): their role is to absorb the luminous energy at a wavelength where PI is unable to operate and to transfer the excitation to PI In that case, the reaction is defined as a sensitized photo induced polymerization [25].

\section{Scheme 21}

The photo-curing of materials; Bisphenol A (bisa) and 1,4-Cyclohexanedimethanol diglycidyl ether (CHDG) was started by photo Acid Generator (PAG). The chemical structural formulae of PAG and CHDG in monomeric forms are shown as below.

The resulted polymers were varied due to monomeric structures. The rigid aromatic closed ring structure of bisa, resulted in significant values of acoustic velocity-as compared to CHDG which is flexible than PAG. $20 \mathrm{~g}$ of monomers and $4 \mathrm{~g}$ of PAG were mixed to prepare the solution mixture. Mixture was placed under vacuum to degas as all of bubbles were disappeared.

For test analysis, mixture was placed within mold made from polytetraflouroethylene (PTEE) with diameter of $50 \mathrm{~mm}$ and depth of $6 \mathrm{~mm}$. The irradiation of mold was done by a LIDAM scientific ultraviolet UV-Lamp with 368 wavelength and intensity of $5 \mathrm{mw} / \mathrm{cm}^{2}$. To get desired value of thickness, aliquots of solution mixture was cured. Same polymers of bias and CHDG were prepared by following methodology. Samples of bias ( $49 \%$ excess) were passed through heating at $333 \mathrm{k}$ to decrease the viscosity and also to remove the air. Filled samples were prepared by two different particles. Materials with less impedance was prepared using Expancel PVDF hollow microsphere with diameter of $60 \times 10^{-6} \mathrm{~m}$ and $60 \mathrm{~kg} / \mathrm{m}^{3}$ density. Then CHDG $(10 \mathrm{~g})$ and PAG $(0.2 \mathrm{~g})$ of expancel were used to prepare the specimens with 16, 27, 36.5, 43.4 and $49 \%$ sphere volume fractions.

Materials with higher impedance was prepared by baso 4 with 4100 $\mathrm{kg} / \mathrm{cm}^{3}$ density and $8-12 \mu \mathrm{m}$ sized particle. Then bisa $(20 \mathrm{~g})$ with PAG $(0.4 \mathrm{~g})$ and $\mathrm{baso}_{4}$ with $13,19,25,31$ and $40 \%$ volume fractions were used to produce specimens. The baso 4 and expancel were achieved from boud materials [26].

\section{Scheme 22}

Different monomers were used as reactive diluents in formulations. By an analysis named photo-differential scanning calorimetry analysis, the results of formulations are studied. Also physical and chemical properties of cured films are studied. In addition, by using DMA technique glass transition temperature and modulus are also determined [27].

\section{Scheme 23}

A methodology (transmission methodology) is described to characterize polymer materials with a range of elastic properties that are suitable for application in piezoelectric transducer and array assemblies. The process permits the fabrication of polymer materials by photocuring thin layers of polymer. The photo-curing method can produce samples of homo-polymers or a blend of homo-polymers. Also, there is no additional amine curing reagent used in photo curing. Furthermore, the methodology described in this paper cures the epoxy resin through opening of the epoxy rings and forming ether linked polymer backbone. The structure of these polymers is therefore very different from those created by the conventional amine cure reaction [26].

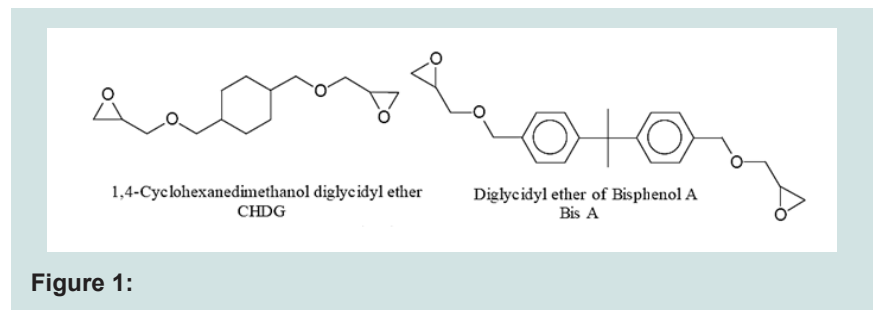

\section{Conclusion}

From the above detailed description, it is clearly resulted that photo curing of ER gave much excellent properties to them. It has enhanced their adhesion properties. It has enhanced their resistance against corrosion.

Photo curing has ceased the chances of environmental pollution created by ER. Due to photo-curing, the thickness and efficiency of ER are also enhanced. After photo curing, ER showed the marvelous working efficacy, eco-friendly compatibility and coloring schematic efficiency.

Photo curing also enhanced the bonding and removing properties of ER. After photo curing, ER protected the material below the coatings of ER. Photo curing also has enhanced the toughness of ER and their compatibility with the environment and also with substrates. Photo curing also enhanced the 3-dimensional property of ER. Shortly, it is stated that photo curing has enhanced the overall properties and working efficiency of ER.

The photo curing is done to enhance the characteristics of ER. Because the photo cured ER are being used in several fields of industry. Mostly, photo cured ER are used in caulking industry, floor making, aircraft making [28], laminate production, aerospace, and composite making [29]. The photo curing has enhanced the strength of ER. It also has enhanced the exceptional flexibility, ability to bond with the other different substrates including metals, excellent adhesion and resistance to many environmental hazards [30].

\section{References}

1. Atif M (2014) Surface modification and characterization of carbon black; UV cured colored epoxy composites.

2. Shen L, Li Y, Zheng J, Lu M, Wu K (2015) Modified epoxy acrylate resin for photocurable temporary protective coatings. Prog Org Coat 89: 17-25.

3. Jin FL, Li X, Park SJ (2015) Synthesis and application of epoxy resin: a review. J Industrial Eng Chem 29: 1-11. 
4. Akutsu F, Inoki M, Daicho N, Kasashima Y, Shiraishi N, et al. (1998) Curing behavior and properties of epoxy resins cured with the diamine having the quinoxaline or triazine structure. J Appl Polym Sci 69: 1737ロ1741.

5. Hamerton I, Hay JN, Howlin BJ, Jepson P, Mortimer S (2001) The development of controllable complex curing agents for epoxy resins. I. Preparation, characterization, and storage behavior of transition metal-diamine complexes. J Appl Polym Sci 80: 1489 1503.

6. Park Sj, Jin FL (2012) Epoxy resins: fluorine systems. Wiley Encyclopedia of Composites $\left(2^{\text {nd }} e d n\right)$

7. Atif M, Yang J, Yang H, Jun N, Bongiovanni R, et al. (2015) Effect of novel UV-curing approach on thermo-mechanical properties of colored epoxy composites in outsized dimensions. J Compos Mater 50: 3147-3156.

8. Ortiz RA, Valdez AG, Padilla EG, Flores RA, Munoz JE (2016) Development of a photocurable glass fiber reinforced epoxy-amine/thiol-ene composites. J Polym Res 23: 30

9. Barletta M, Vesco S, Puopolo M, Tagliaferri V (2015) Graphene reinforced UV-curable epoxy resins: design, manufactureand material performance. Prog Org Coat 90: 414-424

10. Atif M, Bongiovanni R, Yang J (2015) Cationically UV-cured epoxy composites. Polym Rev 55: 90-106.

11. Gallego MM, Manchado MAL, Calza P, Roppolo I, Sangermano M (2015) Gold functionalized graphene as conductive filler in UV-curable epoxy resin. J Mater Sci 50: 605-610.

12. Jiao C, Dong J, Zhang C, Zhuo J, Chen X (2014) Synthesis and properties of a phosphate ester as curing agent in an epoxy resin system. Iran Polym J 23: 591-598.

13. Luangtriratana $P$, Kandola B, Ebdon J (2014) UV-polymerisable, phosphorus containing flame-retardant surface coatings for glass fibre-reinforced epoxy composites. Prog Org Coat 78.

14. Sangermano M, Naguib M, Messori M (2013) Fracture toughness enhancement of UV-cured epoxy coatings containing $\mathrm{Al}_{2} \mathrm{O}_{3}$ nanoparticles. Macromol Mater Eng 298: 1184-1189.

15. Sangermano M, Sordo F, Giovine M, Kortaberria G (2013) UV-cured epoxyzno composites: preparation and characterization. Macromol Mater Eng 298 1304-1308.

16. Roppolo I, Debasu ML, Ferreira RA, Rocha J, Carlos LD, et al. (2013) Photoluminescent epoxy/ $\mathrm{Gd}_{2} \mathrm{O}_{3}: \mathrm{Eu}^{3+} \mathrm{UV}$-cured nanocomposites. Macromol Mater Eng 298: 181-189.
17. Santos MN, Opelt CV, Pezzin SH, Amico SC, Costa CE, et al. (2012) Nanocomposite of photocurable epoxy-acrylate resin and carbon nanotubes: dynamic-mechanical, thermal and tribological properties. Mater Res 16: 367-374.

18. Gallego MM, Hernandez M, Lorenzo V, Verdejo R, Manchado MA, et al. (2012) Cationic photocured epoxy nanocomposites filled with different carbon fillers. Polymer 53: 1831-1838.

19. Sangermano M (2012) Advances in cationic photo polymerization. Pure Appl Chem 84: 2089-2101.

20. Llena M, Maria J, Matia C, Maria A, Juan R, et al. (2011) Effect of a hyperbranched polymer over the thermal curing and the photocuring of an epoxy resin. J Therm Anal Calorim 105: 479-488.

21. Gallego MM, Verdejo R, Manchado MA, Sangermano M (2011) Epoxygraphene UV-cured nanocomposites. Polymer 52: 4664-4669.

22. Decker C, Moussa K (1989) Real-time kinetic study of laser induce polymerization. Macromol 22: 4455-4462.

23. Crivello, James V (2002) Advanced curing technologies using photo-and electron beam induced cationic polymerization. Rad Phy Chemistry 63: 21-27.

24. Sangermano M, Pegel S, Potschke P, Voit B (2008) Antistatic epoxy coatings with carbon nanotubes obtained by cationic photopolymerization. Macromol Rapid Commun 29: 396-400.

25. Fouassier JP, Allonas X, Burget D (2003) Photopolymerization reactions under visible lights: principle, mechanisms and examples of applications. Progr Org Coat 47: 16-36.

26. Troge A, O'Leary RL, Hayward G, Pethrick RA, Mullholland MA (2010) Properties of photocured epoxy resin materials for application in piezoelectric ultrasonic transducer matching layers. J Acoust Soc Am 128: 2704-2714.

27. Kardar P, Ebrahimi M, Bastani S (2013) Curing behavior and mechanica properties of pigmented UV-curable epoxy acrylate coatings. Pigm Resin Tech 43:177-184.

28. Tam LH, Lau D (2014) A molecular dynamics investigation on the crosslinking and physical properties of epoxy-based materials. RSC Advances 62.

29. Park C, Ounaies Z, Watson KA, Pawlowski K, Lowther SE, et al. (2001) Polymer-single wall carbon nanotube composites for potential spacecraft applications. ICASE Report 706: 1-11.

30. Izabel RV, Liane LF, Dimitrios S (1999) Physical aging behavior of a DGEBA DDM system studied by thermal analysis (DSC/DMTA). Polímeros 9: 58-64. 